\title{
Improved Coding-Efficiency Two-Level Source Encoder for RF Switch-Mode Power Amplifiers
}

\author{
Tomasz Podsiadlik, John Dooley, and Ronan Farrell
}

\begin{abstract}
A $\Sigma \Delta$-driven RF switch-mode power amplifier is inherently linear only when it has a two-level output. At the same time, a two-level output generates the largest amount of quantization noise. This brief analyzes the effect of nonequal level spacing in a three-level $\Sigma \Delta$ output and provides a method for shaping the corresponding error noise to regions outside the band of interest. Subsequently, the nonlinearity-shaping property is utilized to obtain an improved two-level drive signal based on three-level $\Sigma \Delta$ modulation. The new binary drive signal is proven to have better adjacent channel leakage ratio and higher coding efficiency than a conventional two-level $\Sigma \Delta$ modulator. In the investigated case, measured coding efficiency improves from $8.9 \%$ of the conventional two-level modulator to $21 \%$ of the modified two-level modulator.
\end{abstract}

Index Terms-Coding efficiency, sigma delta, switch-mode power amplifier (SMPA).

\section{INTRODUCTION}

$\mathbf{S}$ WITCH-MODE operation for RF signal power amplifiers (PAs) has gained interest in recent years. A class-S PA consists of three major blocks: a sigma-delta $(\Sigma \Delta)$ modulator that encodes the input RF signal into a pulse stream, a switch-mode PA (SMPA), and a bandpass filter (Fig. 1) [1], [2]. The encoded pulse stream RF input drives the transistors in SMPA into on and off regions, offering potentially $100 \%$ power efficiency. The drive signal can be either two, three, or more levels. The major reason for the use of a multilevel quantizer in $\Sigma \Delta$ is that the mean square value of the quantization error is proportional to the quantizer level spacing $\Delta$ by $e_{\mathrm{rms}}^{2}=\Delta^{2} / 12$. The more levels are, the less noise is generated. Since $q_{\mathrm{rms}}^{2} \propto e_{\mathrm{rms}}^{2}$, this, in turn, affects the coding efficiency [1] of the source encoder defined by

$$
\eta=\frac{x_{\mathrm{rms}}^{2}}{q_{\mathrm{rms}}^{2}}
$$

where $x_{\mathrm{rms}}^{2}$ denotes the in-band power of a complex signal, i.e., the power recovered after bandpass filtering and, $q_{\mathrm{rms}}^{2}$ is the total power of the output pulse stream. The design of a linear multilevel SMPA operating at gigahertz speed imposes

Manuscript received September 30, 2011; revised April 20, 2012; accepted August 10, 2012. Date of publication September 14, 2012; date of current version October 12, 2012. This work was supported in part by Science Foundation Ireland under Grant 03/CE3/1405 and in part by Bell Labs, Ireland. This brief was recommended by Associate Editor L. Yao.

The authors are with the Centre for Telecommunications Research, National University of Ireland Maynooth, Maynooth, Ireland (e-mail: tpodsiadlik@ eeng.nuim.ie)

Color versions of one or more of the figures in this brief are available online at http://ieeexplore.iee.org.

Digital Object Identifier 10.1109/TCSII.2012.2213432

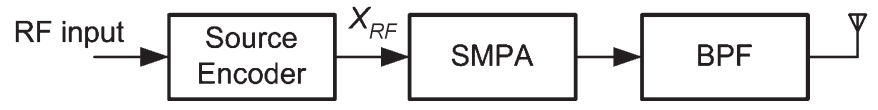

Fig. 1. Class-S PA.

serious implementation challenges concerning linearity. A twolevel $\Sigma \Delta$-driven SMPA is inherently linear and is therefore often preferred over a multilevel SMPA [3].

A few variations of $\Sigma \Delta$ modulator-based source encoders can be distinguished. A common technique utilizes direct bandpass $\Sigma \Delta(B P \Sigma \Delta)$ modulator. The $B P \Sigma \Delta$ modulator's input is fed with a complex signal at a carrier frequency $f_{C}$ with $f_{C} \epsilon\left(f_{\Sigma \Delta} / 4 ; f_{\Sigma \Delta} / 3\right)$ [1], [4]. The major implementation challenge for the direct method is in achieving a sampling rate in a reconfigurable modulator three to four times higher than a carrier frequency, e.g., for $f_{C}=1 \mathrm{GHz}$, the corresponding sampling rate of the $\Sigma \Delta$ modulator is $f_{\Sigma \Delta}=4 \mathrm{GHz}$.

In the other method, the input RF waveform is split into two independent paths consisting of baseband signals $I$ and $Q$. After performing low-pass $\Sigma \Delta(L P \Sigma \Delta)$ modulation, these outputs undergo upconversion and quadrature mixing [5], [6]. As a result, the sampling rate of a $\Sigma \Delta$ modulator several times lower than the carrier frequency is possible. Both of these methods can be used for encoding the input signal. Apart from the difference in sampling rates of the $\Sigma \Delta$ and, therefore, signal-to-noise ratios (SNRs), both offer very similar coding efficiencies. When their outputs are binary, then, the resulting coding efficiency is low, often less than $10 \%$ [1]. The importance of coding efficiency is seen in [4], where it was demonstrated that a three-level SMPA can have better drain efficiency than the equivalent SMPA driven by two-level pulse stream.

In this brief, a nonzero carrier SMPA performance will be examined for its dependence on nonlinearity due to unequal spacing between levels in its three-level output. A method of minimizing the performance degradation is proposed in Section II. In Section III, an improved two-level source encoder for an RF SMPA is derived. Section IV validates the concept providing measured results. Conclusions are provided in Section V.

\section{Nonlinear Three-Level Stage}

The advantage of a three-level $\Sigma \Delta$ modulator over a twolevel modulator is a reduction in the quantization noise power level. The problem arises however when the stage that follows the three-level modulator induces unequal spacings between the levels, causing a nonlinearity and thus degrading SNR. On 
(a)

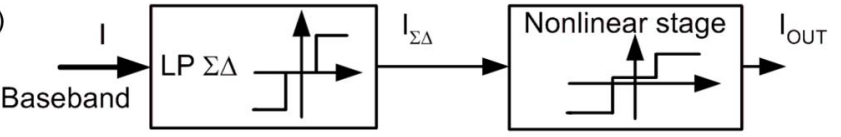

(b)

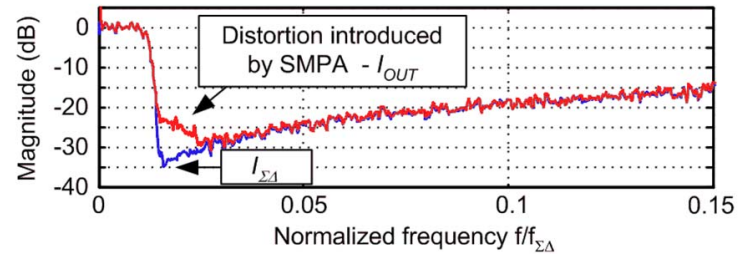

Fig. 2. (a) $\Sigma \Delta$ driving nonlinear three-level SMPA. (b) Output spectrum of the nonlinear SMPA. (a)

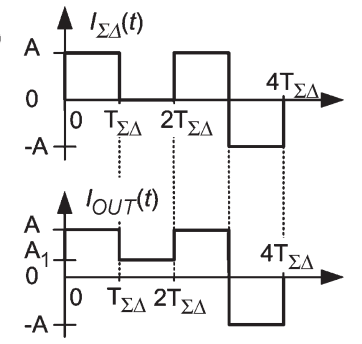

(b)
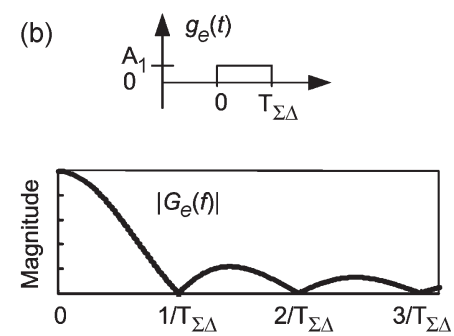

Fig. 3. (a) Input-output waveforms of the nonlinear stage. (b) Error pulse $g_{e}(t)$ and its Fourier transform $\left|G_{e}(f)\right|$.

the other hand, a two-level modulator is inherently linear. It is demonstrated in the following pages that this nonlinearity effect on SNR in the three-level modulator's output can be significantly reduced.

Consider a three-level low-pass $\Sigma \Delta$ modulator followed by a nonlinear stage, e.g., three-level SMPA, shown in Fig. 2(a), whose input is supplied with a baseband signal $I$. The nonlinearity of the SMPA results in a distorted frequency response as shown in Fig. 2(b).

The nonlinearity of the three-level stage can be represented by offsetting the zero output level by some constant value $A_{1}$. For simplicity, assume that the positive and negative values in the output pulse stream of the $\Sigma \Delta$ are the same as in the output from the nonlinear stage, i.e., $\pm A$. The output $I_{\text {OUT }}$ is created by adding a square pulse $g_{e}(t)$ of an amplitude $A_{1}$ to $I_{\Sigma \Delta}$, each time $I_{\Sigma \Delta}$ is zero as shown in Fig. 3(a). If the error $g_{e}(t)$ is an ideal square pulse, then its Fourier transform $G_{e}(f)$ given by (2) will have zeros at integer multiples of $f_{\Sigma \Delta}$ as shown in Fig. 3(b), where $f_{\Sigma \Delta}=1 / T_{\Sigma \Delta}$ denotes the sampling rate of the modulator

$$
G_{e}(f)=T_{\Sigma \Delta} A_{1} \operatorname{sinc}\left(\frac{\omega T_{\Sigma \Delta}}{2}\right) e^{-j \omega T_{\Sigma \Delta} / 2}
$$

A quadrature mixer scheme followed by nonlinear stage is shown in Fig. 4(a). The blocks $c(t)$ and $s(t)$ have $90^{\circ}$ phaseshifted impulse responses with the same fundamental frequency $f_{C}$ as shown in Fig. 4(b). The quadrature mixer supplied by the three-level $I_{\Sigma \Delta}$ and $Q_{\Sigma \Delta}$ outputs generates a complex output signal spectrum at the carrier frequency $f_{C}$ similar to the spectrum shown in Fig. 6(b). It is noticed that, when $I_{\Sigma \Delta}=Q_{\Sigma \Delta}=0$, the quadrature mixer output becomes $x_{\mathrm{RF}}=$ 0 , while the corresponding output from the nonlinear stage is $x_{\mathrm{RF}}^{\prime}=A_{1}=g_{e}(t)$; this situation is shown in Fig. 5(a) and (b). (a)

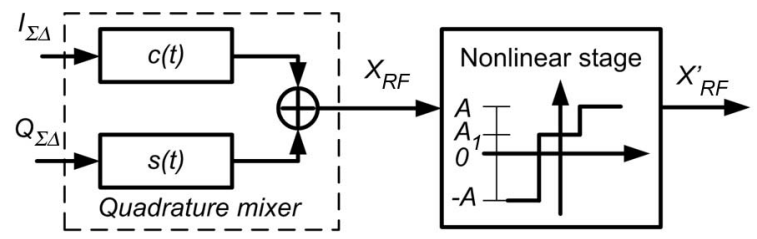

(b)
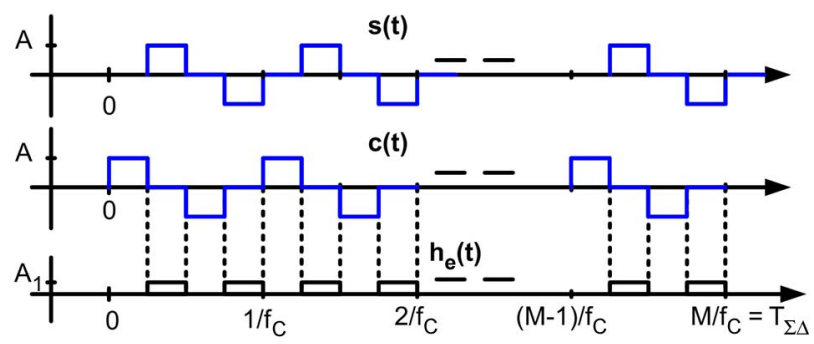

Fig. 4. (a) Quadrature mixer followed by nonlinear stage. (b) Impulse responses of quadrature mixer $c(t)$ and $s(t)$ and error pulse $h_{e}(t)$ corresponding to $I_{\Sigma \Delta} \neq 0$ and $Q_{\Sigma \Delta}=0$

(a)

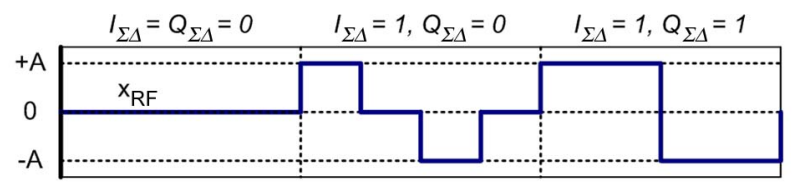

(b)

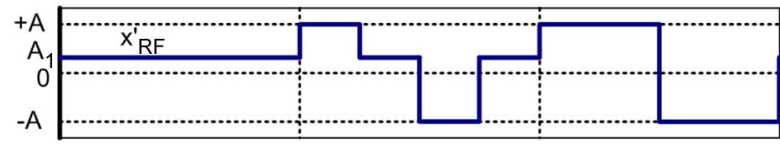

(c)

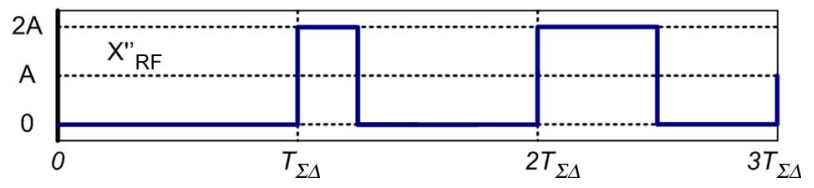

Fig. 5. Time-domain waveforms for $M=1$ and $f_{C}=f_{\Sigma \Delta}$. (a) Linear modulator's output $x_{\mathrm{RF}}(t)$. (b) Nonlinear $x_{\mathrm{RF}}^{\prime}(t) ; A_{1}=0.2 A$. (c) Nonlinear $x_{\mathrm{RF}}^{\prime \prime}(t)=x_{\mathrm{RF}}^{\prime}(t)+A ; A_{1}=-A$.

The error that occurs due to the $A_{1}$ offset in the nonlinear stage is shaped in frequency domain by function $G_{e}(f)$.

The quadrature mixing operation of the $\Sigma \Delta$-encoded baseband signals $I_{\Sigma \Delta}$ and $Q_{\Sigma \Delta}$ can be expressed by

$$
x_{\mathrm{RF}}(t)=I_{\Sigma \Delta} \cdot \cos \left(2 \pi f_{C} t\right)+Q_{\Sigma \Delta} \cdot \sin \left(2 \pi f_{C} t\right) .
$$

If, in the course of the mixing, a carrier $f_{C}$ such that

$$
f_{C}=M f_{\Sigma \Delta}
$$

is chosen with $M$ being a positive integer number, then the complex signal carrier frequency coincides with a zero of $G_{e}(f)$. The error in the $X_{\mathrm{RF}}^{\prime}(f)$ corresponding with the nonlinearity is suppressed at $f_{C}$, and a spectral separation between the signal of interest and the error is provided.

Two types of error pulse are distinguished when $I_{\Sigma \Delta}$ and $Q_{\Sigma \Delta}$ are three-level signals.

1) Both are zero, i.e., $I_{\Sigma \Delta}=Q_{\Sigma \Delta}=0$, yielding an error pulse $g_{e}(t)$ as described earlier.

2) Only one is zero, i.e., $I_{\Sigma \Delta} \neq 0$ and $Q_{\Sigma \Delta}=0$, or $I_{\Sigma \Delta}=0$ and $Q_{\Sigma \Delta} \neq 0$, yielding an error pulse denoted by $h_{e}(t)$.

In the case of 2), the error pulse $h_{e}(t)$ is created by offsetting zero-valued intervals in an impulse response of a sine or a 
(a)

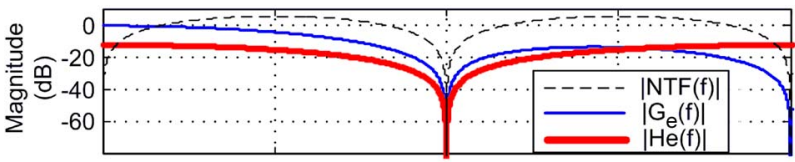

(b)

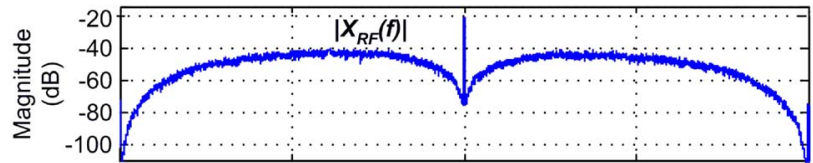

(c)

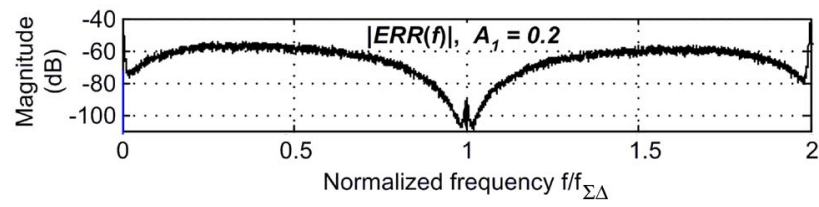

Fig. 6. (a) First-order noise transfer function, $\left|G_{e}(f)\right|$, and $\left|H_{e}(f)\right|$. (b) Linear three-level output spectrum $\left|X_{R F}(f)\right|$. (c) Error's spectrum $|E R R(f)|$, for $A_{1}=0.2 A$.

cosine function by the constant $A_{1}$ as it is shown in Fig. 4(b). Computing the Fourier transform of $h_{e}(t)$ yields (5), a function that has a zero at $f_{C}=M f_{\Sigma \Delta}$

$$
\left\{\begin{array}{l}
H_{e}(f)=2 A_{1} \cdot \frac{T_{\Sigma \Delta}}{8 M} \cdot \operatorname{sinc}\left(\omega \frac{T_{\Sigma \Delta}}{8 M}\right) \sum_{m=1}^{2 M} e^{-j \omega t_{m}} \\
t_{m}=\frac{T_{\Sigma \Delta}}{8 M}[1+4(m-1)] .
\end{array}\right.
$$

The occurrence of the error of types 1) and 2) can be written as a convolution of error signals $e_{1}[n]$ and $e_{2}[n]$ with pulses $g_{e}(t)$ and $h_{e}(t)$ and added to the linear output $x_{\mathrm{RF}}(t)$ producing the output of the nonlinear stage

$$
x_{\mathrm{RF}}^{\prime}(t)=x_{\mathrm{RF}}(t)+e_{1}[n] \star g_{e}(t)+e_{2}[n] \star h_{e}(t) .
$$

When (6) is rewritten in frequency domain, it yields

$$
X_{\mathrm{RF}}^{\prime}(f)=X_{\mathrm{RF}}(f)+E_{1}(f) G_{e}(f)+E_{2}(f) H_{e}(f)
$$

since

$$
G_{e}\left(f_{C}=M f_{\Sigma \Delta}\right)=H_{e}\left(f_{C}=M f_{\Sigma \Delta}\right)=0
$$

then

$$
X_{\mathrm{RF}}^{\prime}\left(f_{C}\right)=X_{\mathrm{RF}}\left(f_{C}\right) .
$$

The output $X_{\mathrm{RF}}^{\prime}$ remains unaffected by the nonlinearity of the SMPA at the carrier $f_{C}$.

Waveforms of an ideal $x_{\mathrm{RF}}$ and $x_{\mathrm{RF}}^{\prime}$ for the case of $M=1$, $A=1$, and $A_{1}=0.2$ are drawn in Fig. 5(a) and (b). The signal's spectrum appears at a carrier $f_{C}=1$, which coincides with zeros of $G_{e}(f)$ and $H_{e}(f)$ as shown in Fig. 6(a) and (b). The quantization noise from the $\Sigma \Delta$ modulator shaped by an $N T F$ and the error due to the $A_{1}$ offset are suppressed at the carrier frequency. The linear output $X_{\mathrm{RF}}(f)$ and the error spectrum found by computing the FFT of $\operatorname{err}(t)=e_{1}[n]$ 太 $g_{e}(t)+e_{2}[n] \star h_{e}(t)$ are shown in Fig. 6(b) and (c). For values of $A=1$ and $A_{1}=0.2$, the introduced error magnitude at $f_{C}$ is $15 \mathrm{~dB}$ below the quantization error magnitude of the threelevel first-order $\Sigma \Delta$ modulator pair as shown in Fig. 6(b). This indicates only a slight degradation of the performance in the band of interest $\left(f_{C}\right)$ of the nonlinear output $X_{\mathrm{RF}}^{\prime}(f)$.

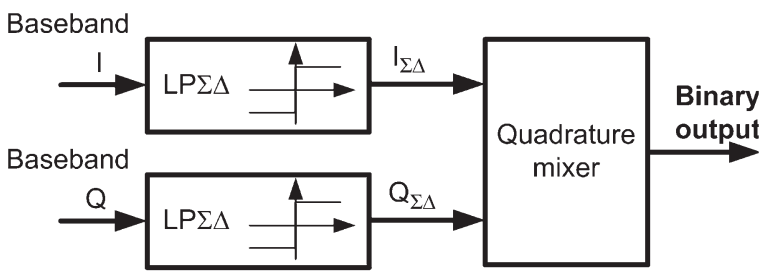

Fig. 7. Conventional two-level signal modulator.

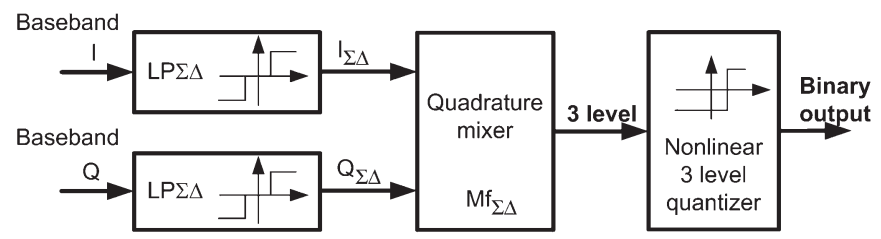

Fig. 8. Modified two-level signal modulator.

\section{Modified Two-Level Source Encoder}

The property of spectral shaping of the error induced by the nonlinearity in a three-level quantizer explained in Section II can be deployed to modify a conventional two-level drive signal for RF SMPA.

Often, a binary $\Sigma \Delta$ modulator's output switches between zero and some nonzero-valued amplitude, assumed here as 0 and $+2 A$ such as the waveform shown in Fig. 5(c). This pulse stream can drive a two-level class-D PA [1], [4]. This drive signal can be generated with the use of the conventional modulator, employing a pair of two-level $\Sigma \Delta$ modulators shown in Fig. 7. The coding efficiency in this case is, however, poor. The conventional modulator whose two output levels are 0 and $+2 A$, supplied with an input of a mean value of $+A$, will be delivering an output level of $+2 A$ for half of the operation time regardless of the input signal's power. The power of such output pulse stream is given by

$$
q_{2 L}^{2}=2 A^{2}=\text { const. }
$$

The proposed modulator structure is shown in Fig. 8. It is created by replacing the pair of two-level $\Sigma \Delta$ modulators with a pair of three-level ones in the conventional modulator. Next, the quadrature mixer is followed by nonlinear three-level quantizer whose middle level is offset by $A_{1}=-A$, forcing it to the same value as the lower level. Thus, the three-level signal $x_{\mathrm{RF}}(t)$ in Fig. 5(a) transforms to a two-level pulse stream. Adding a dc offset of $A$ to this signal yields the signal $x_{\mathrm{RF}}^{\prime \prime}(t)$ in Fig. 5(c).

The spectral separation of the wanted signal at $f_{C}$ to the error generated by the nonlinearity of the quantizer is guaranteed by ensuring that a positive integer $M$ value is used for the quadrature mixer as described in Section II and (7)-(9). The resulting two-level output of the proposed modulator has outof-band noise power level close to a noise power level of the three-level modulator, slightly degraded by nonlinearity in the quantizer.

Simulated output spectra of both conventional and proposed two-level modulators for the case of $M=1, O S R=80$, and $\operatorname{NTF}(z)=1-z^{-1}$ are shown in Fig. 9. Reduction of the error magnitude in adjacent channels can be seen for the modified 


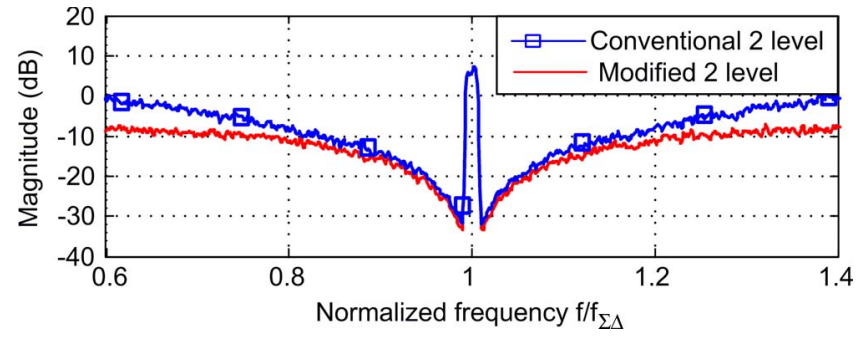

Fig. 9. Simulated output spectra of conventional and modified first-order $\Sigma \Delta$ modulators for $M=1$ and $O S R=80$.

two-level modulator versus conventional two-level modulator. Both modulators use the same sampling rate $\left(f_{\Sigma \Delta}=1\right)$.

\section{A. Coding Efficiency of the Binary Drive Signal}

A three-level, low-pass $\Sigma \Delta$ modulator whose output can take one of three values: $-A, 0$ or $+A$ and $A=1$, is supplied by an input signal $I$, with mean value $\bar{I}=0$. The mean absolute value in such a $\Sigma \Delta$ output is close to the mean absolute of its input, i.e., $\overline{|I|} \approx \overline{\left|I_{\Sigma \Delta}\right|}$. Provided that $I$ and $Q$ have the same mean absolute values, i.e., $\overline{|I|}=\overline{|Q|}$, after performing quadrature mixing, the probability of the output being $+A$ is

$$
P_{A} \approx 0.5 \mid \overline{|I|}=0.5 \overline{|Q|} .
$$

After conversion of the three-level waveform $x_{\mathrm{RF}}(t)$ to a two-level $x_{R F}^{\prime \prime}(t)$ shown in Fig. 5(a) and (c), the power of the output pulse train can be estimated from

$$
q_{\mathrm{MOD}}^{2}=P_{A}(2 A)^{2} \approx 2 A^{2}(\overline{|I|})
$$

the coding efficiency of the modified system becomes

$$
\eta_{\mathrm{MOD}} \approx \frac{x_{\mathrm{rms}}^{2}}{2 A^{2}(\overline{|I|})} .
$$

Comparing (12) with (10), it is concluded that the modified two-level modulator will always produce less noise power in its output than the conventional two-level modulator; this property is regardless of parameter $M$

$$
2 A^{2}(\overline{|I|})<2 A^{2} .
$$

Simulated coding efficiency versus peak-to-average power ratio (PAPR) of a band-limited input is shown in Fig. 10. While the coding efficiency of the conventional two-level system decreases toward zero with an increase of PAPR, the modified two-level pulse stream maintains a coding efficiency of approximately $8 \%$, even for large PAPR values.

\section{B. ACLR and SNR in the Modified Two-Level Modulator's Output}

The input signal used for adjacent channel leakage ratio (ACLR) and SNR simulations was four symbol quadrature amplitude modulated (4QAM) signal with $P A P R=8 \mathrm{~dB}$, and the noise transfer function of $\Sigma \Delta$ modulator was $N T F=$ $1-z^{-1}$. The ACLR performance was calculated for an $O S R$

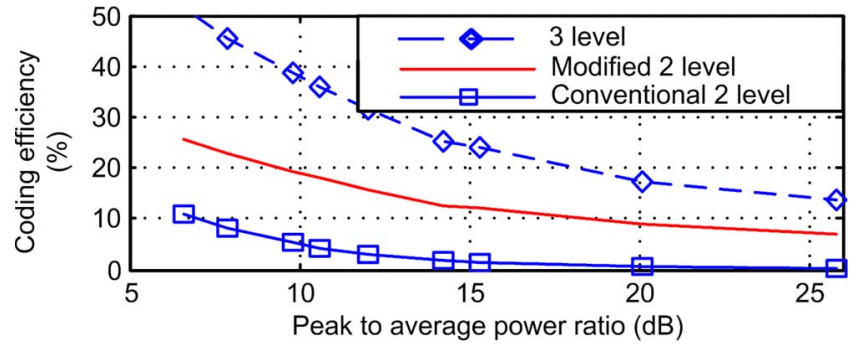

Fig. 10. Simulated coding efficiency for $O S R=80, M=1$, and $N T F=$ $1-z^{-1}$.

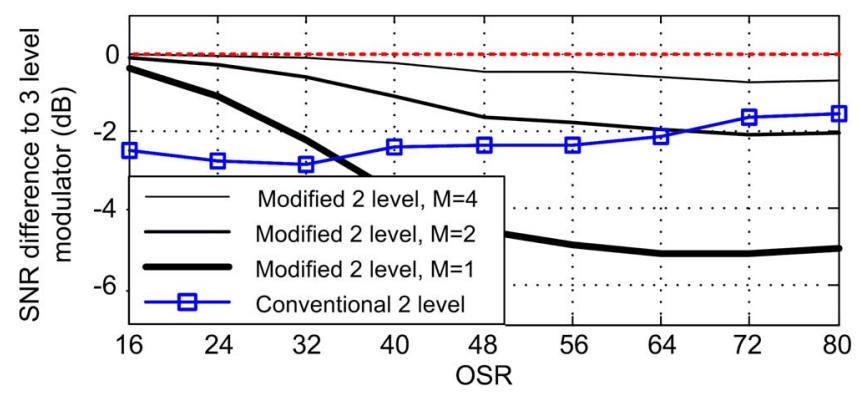

Fig. 11. Simulated SNR versus OSR with $N T F=1-z^{-1}$.

varying from 16 to 80 , and it showed almost constant difference between the three-level, modified two-level, and the conventional two-level modulators. The modified two-level modulator's ACLR performance was worse than that of the three-level system by approximately $0.8 \mathrm{~dB}$, and it showed $2 \mathrm{~dB}$ of an improvement in comparison to the conventional twolevel modulator.

Although the modified two-level modulator exhibits a lower error power level in the adjacent channels compared to the conventional two-level modulator, this is not so for an in-band error power. Fig. 11 shows the SNR of two-level systems below the SNR of three-level modulator, e.g., $0 \mathrm{~dB}$ indicates that a two-level system provides the same SNR as the three-level one, while $-1 \mathrm{~dB}$ means $1-\mathrm{dB}$ SNR degradation compared with the three-level modulator.

The $O S R=80$ indicates that calculation is carried out only inside the signal band, while for lower values of OSR, also an out-of-band noise power is included in calculations.

It is observed that, when $M=1$, the modified two-level modulator exhibits SNR nearly as good as the SNR of a threelevel modulator at low $O S R$ values. The SNR degrades with an increase of $O S R$, performing worse than conventional two-level modulator when $O S R$ increases above 35. This implies that, for $M=1$, the modified two-level modulator generates more of an in-band error power than the conventional two-level one.

In contrast, SNR improvement in the output of the modified modulator is observed when $M=4$. The SNR performance in this case is better than that of the conventional two-level modulator over the entire displayed range of $O S R$.

The additional error term in the modified modulator's output is shaped in a frequency domain by $G_{e}(f)$ and $H_{e}(f)$. Fig. 3(b) shows that, the higher frequency zero in $G_{e}(f)$ is chosen for a carrier frequency, the lower the magnitude of $G_{e}(f)$ is, e.g., $\left|G_{e}(f)\right|$ is lower in proximity of $f_{C}=2 / T_{\Sigma \Delta}$ than in proximity of $f_{C}=1 / T_{\Sigma \Delta}$. The same property applies to $H_{e}(f)$; as a 
(a)

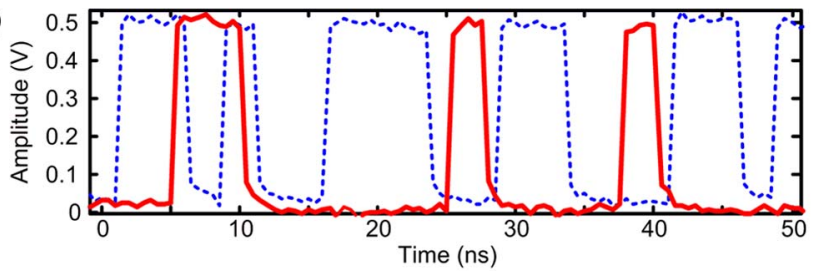

(b)

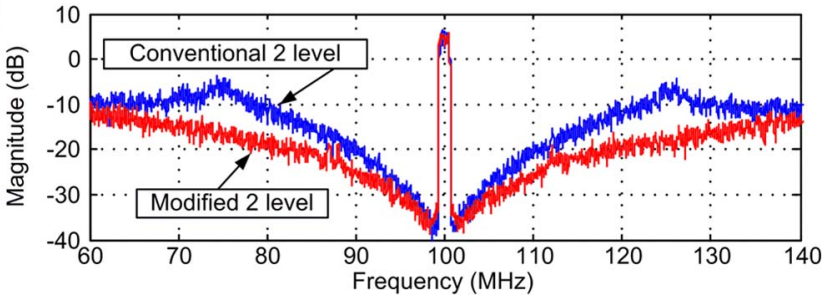

Fig. 12. (a) Measured time-domain waveforms: (Dashed line) Conventional two-level modulator and (continuous line) modified modulator. (b) Modulators' output spectra.

result, the $S N R$ in the output from the modified modulator improves when $M$ increases.

\section{Measured Results and Discussion}

The conventional two-level system of Fig. 7 and the modified two-level modulator of Fig. 8 have been implemented in Virtex II Pro field-programmable gate array (FPGA) board.

The implementation of the modified two-level modulator requires minor additional overhead for hardware in comparison with the conventional two-level modulator. When implemented, the use of a three-level quantizer instead of a two-level quantizer increased the hardware count by an addition of four logic gates. Also, several AND gates-depending on the $M$ parameter-were sufficient to convert a three-level output to a binary form. No significant power consumption difference between the conventional and the modified modulators should therefore be observed.

Each $\Sigma \Delta$ modulator was provided with a $0.625-\mathrm{MHz}$-wide baseband $I, Q$ input, creating 4QAM signal with a $P A P R$ of $8 \mathrm{~dB}$. The same input was used for measuring the output spectra of the conventional two-level and the modified modulators. Both $\Sigma \Delta$ modulators were first orders with the same noise transfer function $N T F=1-z^{-1}$ and sampled at the rate of $f_{\Sigma \Delta}=100 \mathrm{MHz}$. For $M=1$, the corresponding carrier signal was $f_{C}=100 \mathrm{MHz}$, resulting in the output pulse stream bit rate of $400 \mathrm{MHz}$. A 20-GS/s digital oscilloscope was used for measuring the output from the FPGA board.

It is observed from the measured time-domain waveforms, shown in Fig. 12(a), that the total duration of high state output is shorter in the modified modulator's case, which indicates that the pulse train produced by the modified modulator has lower power than the output of the conventional modulator, i.e., $q_{\text {MOD }}^{2}<q_{2 L}^{2}$. The output spectra of both systems are shown in Fig. 12(b). Matching with the expectation, the modified system generates less quantization noise than the conventional twolevel modulator. Although both modulators were characterized by the same $N T F$, a difference in the noise shaping occurred between the two of them. Maxima of the noise power of the conventional two-level modulator at $f=75 \mathrm{MHz}$ and $f=$ $125 \mathrm{MHz}$ were caused by the input signal used. This did not occur in the modified modulator's output due to the use of threelevel quantizers in $L P \Sigma \Delta$ modulators. A slight noise level increase near the carrier frequency in the output spectrum of modified system is observed.

There are two sources of this performance degradation. First is caused by an error pulse shaping different from the ideal described by $g_{e}(t)$ and $h_{e}(t)$. The difference results from the use of a nonideal digital-to-analog converter in FPGA. It was also observed that the increase of an adjacent channel noise in the output from the modified two-level modulator is somewhat correlated with relatively low PAPR of an input signal.

Measured output samples were used for determining coding efficiency. Input signals to both conventional and modified modulators were characterized by $\overline{|I|}=\overline{|Q|}=0.091 \mathrm{~V}$, with an average power of $x_{\mathrm{rms}}^{2}=0.011 \mathrm{~V}^{2}$ and an amplitude peaking between $\pm 0.25 \mathrm{~V}$. Equations (10) and (12) can be used to calculate the expected power in the output pulse trains, i.e., $q_{\mathrm{MOD}-\mathrm{CALC}}^{2} \approx 0.0455 \mathrm{~V}^{2}$ and $q_{2 \mathrm{~L}-\mathrm{CALC}}^{2}=0.125 \mathrm{~V}^{2}$. This is close to the measured values of $q_{\mathrm{MOD}}^{2}=0.053 \mathrm{~V}^{2}$ and $q_{2 L}^{2}=0.124 \mathrm{~V}^{2}$. Coding efficiencies for the measured signals computed with (1) are $\eta_{2 L}=8.9 \%$ and $\eta_{\mathrm{MOD}}=21 \%$.

\section{CONCLUSION}

In this brief, a new approach to signal modulation for an RF SMPA has been described. It has been demonstrated that, in certain cases, an error introduced by the nonlinearity due to unequally spaced levels in a three-level output can be efficiently minimized within a signal band. The mechanism of spectral shaping of the introduced error has been exploited to derive an improved-coding-efficiency two-level modulator. The modified two-level modulator has been implemented and demonstrated for a complex signal at $100-\mathrm{MHz}$ carrier frequency. The measured results show coding efficiency improvement from $8.9 \%$ in a conventional two-level approach to $21 \%$ for the modified two-level system. As shown in Fig. 12, the modified twolevel modulator offers significant noise power reduction in a wideband centered on the signal frequency, with little impact on signal quality and with low implementation complexity.

\section{REFERENCES}

[1] T. Johnson and S. P. Stapleton, "RF class-D amplification with bandpass sigma-delta modulator drive signals," IEEE Trans. Circuits Syst. I, Reg. Papers, vol. 53, no. 12, pp. 2507-2520, Dec. 2006.

[2] A. Wentzel, C. Meliani, and W. Heinrich, "RF class-S power amplifiers: State-of-the-art results and potential," in Proc. IEEE MTT-S Int. MTT, May 23-28, 2010, p. 1.

[3] J. Choi, J. Yim, J. Yang, J. Kim, J. Cha, D. Kang, D. Kim, and B. Kim, "A $\Delta \Sigma$-Digitized polar RF transmitter," IEEE Trans. Microw. Theory Tech., vol. 55, no. 12, pp. 2679-2690, Dec. 2007.

[4] T.-P. Hung, J. Rode, L. E. Larson, and P. M. Asbeck, "Design of H-bridge class-D power amplifiers for digital pulse modulation transmitters," IEEE Trans. Microw. Theory Tech., vol. 55, no. 12, pp. 2845-2855, Dec. 2007.

[5] A. Frappe, A. Flament, B. Stefanelli, A. Kaiser, and A. Cathelin, "An all-digital RF signal generator using high-speed modulators," IEEE J. Solid-State Circuits, vol. 44, no. 10, pp. 2722-2732, Oct. 2009.

[6] M. Helaoui, S. Hatami, R. Negra, and F. M. Ghannouchi, "A novel architecture of delta-sigma modulator enabling all-digital multiband multistandard RF transmitters design," IEEE Trans. Circuits Syst. II, Exp. Briefs, vol. 55, no. 11, pp. 1129-1133, Nov. 2008. 